\title{
Influence of edge effects on laser-induced surface displacement of opaque materials by photothermal interferometry
}

Cite as: J. Appl. Phys. 128, 044509 (2020); https://doi.org/10.1063/5.0015996

Submitted: 01 June 2020 . Accepted: 08 July 2020 . Published Online: 29 July 2020

G. A. S. Flizikowski (D), B. Anghinoni (D) J. H. Rohling (D), M. P. Belançon (D), R. S. Mendes, M. L. Baesso, L. C. Malacarne (D), T. Požar (D) S. E. Bialkowski (D), and N. C. C. Astrath (iD)

\section{COLLECTIONS}

Paper published as part of the special topic on Photothermics

Note: This paper is part of the Special Topic on Photothermics.
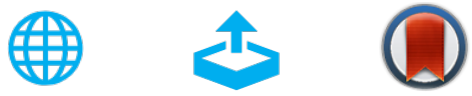

\section{ARTICLES YOU MAY BE INTERESTED IN}

Thermal kinetic inductance detectors for millimeter-wave detection

Journal of Applied Physics 128, 044508 (2020); https://doi.org/10.1063/5.0002413

Photoacoustic imaging for surgical guidance: Principles, applications, and outlook Journal of Applied Physics 128, 060904 (2020); https://doi.org/10.1063/5.0018190

Spatially resolved thermoreflectance techniques for thermal conductivity measurements from the nanoscale to the mesoscale

Journal of Applied Physics 126, 150901 (2019); https://doi.org/10.1063/1.5120310

\section{Lock-in Amplifiers up to 600 $\mathrm{MHz}$}
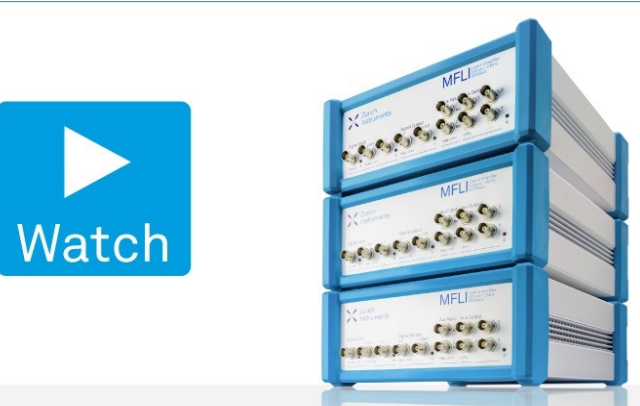


\title{
Influence of edge effects on laser-induced surface displacement of opaque materials by photothermal interferometry
}

Cite as: J. Appl. Phys. 128, 044509 (2020); doi: 10.1063/5.0015996

Submitted: 1 June 2020 - Accepted: 8 July 2020 .

Published Online: 29 July 2020

G. A. S. Flizikowski, ${ }^{1}$ (D) B. Anghinoni, ${ }^{1}$ (D) J. H. Rohling, ${ }^{1}$ (D) M. P. Belançon, ${ }^{2}$ (D) R. S. Mendes, ${ }^{7}$ M. L. Baesso,

L. C. Malacarne, ${ }^{1}$ (D) T. Požar, ${ }^{3}$ (D) S. E. Bialkowski, ${ }^{4}$ (D) and N. G. C. Astrath ${ }^{1, a)}$ (i)

\begin{abstract}
AFFILIATIONS
${ }^{1}$ Departamento de Física, Universidade Estadual de Maringá, Maringá, PR 87020-900, Brazil

${ }^{2}$ Departamento de Física, Universidade Tecnológica Federal do Paraná, Pato Branco, PR 85503-390, Brazil

${ }^{3}$ Faculty of Mechanical Engineering, University of Ljubljana, Ljubljana 1000, Slovenia

${ }^{4}$ Department of Chemistry and Biochemistry, Utah State University, Logan, Utah 84322-0300, USA
\end{abstract}

Note: This paper is part of the Special Topic on Photothermics.

a) Author to whom correspondence should be addressed: ngcastrath@uem.br

\begin{abstract}
We demonstrate the influence of edge effects on the photothermal-induced phase shift measured by a homodyne quadrature laser interferometer and compare the experiments with rigorous theoretical descriptions of thermoelastic surface displacement of metals. The finite geometry of the samples is crucial in determining how the temperature is distributed across the material and how this affects the interferometer phase shift measurements. The optical path change due to the surface thermoelastic deformation and thermal lens in the surrounding air is decoded from the interferometric signal using analytical and numerical tools. The boundary/edge effects are found to be relevant to properly describe the interferometric signals. The tools developed in this study provide a framework for the study of finite size effects in heat transport in opaque materials and are applicable to describe not only the phase shift sensed by the interferometer but also to contribute to the photothermal-based technologies employing similar detection mechanisms.
\end{abstract}

Published under license by AIP Publishing. https://doi.org/10.1063/5.0015996

\section{INTRODUCTION}

The dynamic events following light absorption at the surface of the inspected material contain information on the structure of matter, its geometry, and its physical and thermoelastic properties. The temperature changes resulting from optical absorption are responsible for generating thermoelastic disturbances detectable at the surfaces of the sample, bringing characteristic signatures of defects and shape of the system that can be exploited experimentally in several different ways. External and internal boundaries are accessible in such a system, for example, by monitoring the perturbations caused by heat diffusion at the interfaces ${ }^{1}$ or by elastic waves reverberating within the material. ${ }^{2}$

In practice, the inspected materials have to be prepared as samples of well-defined finite dimensions, often as small as possible, especially when the material is expensive or difficult to be produced.
This, in turn, imposes some limitations on how to model the response of such samples and compare the model to the measurements to retrieve the unknown sample properties. The lateral heat flow confinement or the elastic waves reflections from the samples surfaces are known as edge effects. They become important when the heat diffusion flow is laterally halted or when the disturbances reflect from the edges and return to the detection site. The edge effects are responsible for the scattering of elastic waves propagating inside materials and form the basis of photothermal ultrasound detection systems with applications ranging from biological imaging ${ }^{3,4}$ to defect inspection. ${ }^{5,6}$ Edge effects also have an important role in material processing procedures. ${ }^{7-9}$ Several methods including laser interferometry have been used for investigating surface properties, ${ }^{10,11}$ local magnetic property deteriorations in metals, ${ }^{12}$ microdamage evolution, ${ }^{13}$ fatigue failure, ${ }^{14}$ mechanical properties, ${ }^{15,16}$ and 
as optical image hiding method. ${ }^{17,18}$ This technique reveals how nanostructured surfaces and structures can be characterized, such as textured stainless steel, ${ }^{19}$ Mylar polymer films, ${ }^{20}$ and biomaterials, for instance, polymerized hexamethyldisiloxane ${ }^{21}$ and hydroxyapatite materials. ${ }^{2,23}$ Interferometric methods are also used in all-optical ultrasound detection, ${ }^{24}$ which can overcome some limitations when compared with piezoelectric transducers detection in biological imaging. $^{25,26}$

In many photothermal interferometric methodologies, simplified heat transfer treatments are feasible in applications where the diameter of the sample is much larger than its thickness. This approximation minimizes the edge effects and the system can be modeled properly using (semi)analytically solvable, one-dimensional formulations. On the other hand, finite systems are complex and require three-dimensional numerical description of the heat propagation and the corresponding thermoelastic deformation. Here, we report on the edge effects occurring under laser-induced surface displacements of metals measured by optical interferometry. The interferometer used in this study, the homodyne quadrature laser interferometer (HQLI), has a wide dynamic range and a constant sensitivity. ${ }^{27}$ It is traceable to the primary standard of length ${ }^{28}$ and is thus very accurate in measuring physical displacement of reflective objects as well as changes in the refractive index of transparent gases. This detector is thus both, a displacement-measuring device $^{29}$ and a refractometer, ${ }^{30}$ exactly what is needed to simultaneously probe the surface bending of a heated reflective solid and the temperature rise in the gas above the heated sample, since both information can be encoded into the optical phase difference (OPD). The HQLI has already been used to detect very fast transients (temporal resolution of about $10 \mathrm{~ns}$ ), ${ }^{31}$ where elastic waves play an important role, and also slow phenomena, such as the creeping of polymers (total measuring time of minutes) ${ }^{32}$ and in determination of the absolute gravity. ${ }^{33}$ This makes the HQLI a complementary technique to the thermal mirror (TM) and thermal lens (TL) inspection of materials with a notable difference. ${ }^{34}$ The HQLI is sensitive to the absolute displacements and absolute changes of the refractive index encoded in the OPD, while the TM/TL techniques sense the relative changes, encoded as the wave front distortion of the optical probe beam. The notable advantage of using the HQLI is the capability of probing point-displacements, ${ }^{35}$ while the TM/TL proved to be independent of the environmental vibrations making this detection method more robust. ${ }^{34}$

In this study, the HQLI is used to detect the laser-induced thermoelastic surface displacement in metals under on/off continuous laser excitation. Heat generated by light absorption on the surface of the sample builds up and dissipates within the material and out to the coupling air. The finite geometry of the samples is crucial in determining how the temperature is distributed across the material and how this affects the interferometer phase shift measurements. The optical path change due to the surface thermoelastic deformation and the TL in the surrounding air is decoded from the interferometric signal using semi-analytical and numerical tools. The boundary/edge effects are found to be relevant to properly describe the interferometric signals. The tools developed in this study are applicable to describe not only the phase shift sensed by the interferometer but also contribute to the photothermalbased technologies employing similar detection mechanisms.

\section{THEORY}

Two theoretical approaches were used to model the response of the specimen and the surrounding air to laser heating of the sample surface. The first approach gives a neat analytical solution, often accurate enough to give useful results, especially when the laser beam diameter is small compared to the dimensions of the sample and when the heat diffusion has not yet been affected by the sample boundaries. The second one is a more realistic numerical procedure based on the finite elements analysis (FEA).

In both models, cylindrical symmetry is considered, defined by the coordinates $(r, z, \varphi, t)$, where $r$ is the axial distance from the $z$-axis, $\varphi$ is the azimuth, a problem independent variable, and $t$ is the time. Three fields of interest are calculated: the scalar temperature rise field in the fluid $T_{f}(r, z, t)$ and in the solid sample $T_{s}(r, z, t)$ and the vector displacement field of the sample $\mathbf{u} \equiv \vec{u}(r, z, t)$. These fields are then used to calculate the optical phase difference (OPD) $\Phi(r, t)$, measurable by an appropriate interferometer.

The main difference between the two models is the geometry of the problem. The analytical approach assumes two homogeneous and isotropic half-spaces, a light absorbing solid sample $(z>0)$ in contact at $z=0$ with a non-absorbing fluid $(z<0)$. The FEA model is more realistic, because it additionally takes into account the exact geometry of the sample (a homogeneous and isotropic cylinder with a finite radius and a finite height) as shown in Fig. 1, including the sample mount. Physically, the key novelty introduced by taking the finite lateral dimension of the sample into account is the lateral boundary that prohibits an effective lateral diffusion of heat within the sample.

The surface of the sample is illuminated through the fluid using a temporally modulated [on-off boxcar-modulated continuous wave $(\mathrm{cw})$ with the exposure time $\xi$ ] Gaussian $\left(\mathrm{TEM}_{00}\right)$ laser beam coinciding with the $z$-axis and having a radius of $w_{\mathrm{e}}$ at the surface of the sample, as shown in Fig. 1. Assuming a temperature independent reflectivity at the excitation laser wavelength $R$, the surface reflects a fraction $P R$ of the laser power $P$ and absorbs $P(1-R)$. The absorbed light is converted into heat, which is then diffused into the sample and the neighboring fluid (air). As a consequence of thermal expansion, the surface is deformed according to the temperature profile in the sample. Additionally, a thermal lens is induced

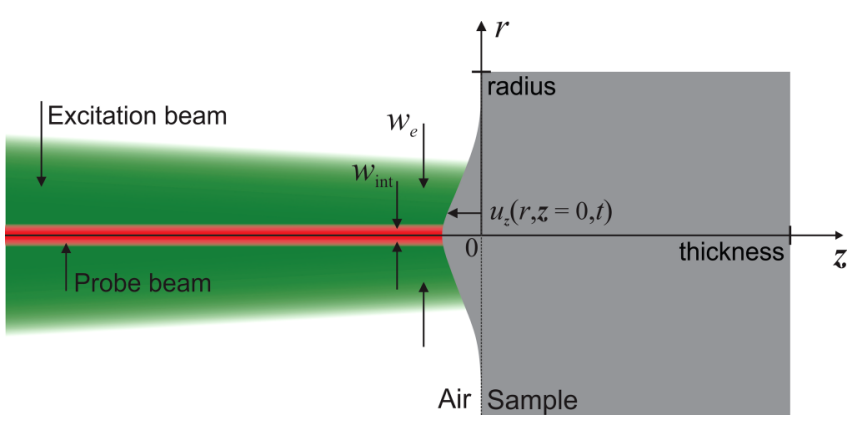

FIG. 1. Geometric scheme of the beams in the measurement arm of the interferometer. 
above the solid sample-the refractive index of the air is temperature dependent. A simultaneous detection of both, the out-of-plane surface displacement and the TL of the air was performed by a HQLI.

\section{A. Analytical approach}

\section{Temperature fields}

The spatiotemporal distribution of the temperature (rise) is sought for the sample and fluid. It is given by the solution of the coupled heat diffusion equations, ${ }^{36}$

$$
\frac{\partial T_{i}(r, z, t)}{\partial t}-D_{i} \nabla^{2} T_{i}(r, z, t)=Q_{i} Q(r) Q(z),
$$

with proper boundary and initial conditions as given in Ref. 37. $D_{i}=k_{i} / \rho_{i} c_{p i}$ is the thermal diffusivity, $k_{i}$ is the thermal conductivity, $\rho_{i}$ is the mass density, and $c_{p i}$ is the specific heat for the sample $(i=s)$ and fluid $(i=f)$. The heat source in the sample is $Q_{s}=2 P(1-R) \phi / \pi c_{s} \rho_{s} w_{\mathrm{e}}^{2}$ and $Q(r)=\exp \left(-2 r^{2} / w_{\mathrm{e}}^{2}\right)$. Since the fluid is assumed not to absorb any laser light, $Q_{f}=0$. The portion of the absorbed power converted into heat is represented by $\phi$, which in this case is 1 as the absorbed power is completely converted into heat. For opaque, highly absorbing materials, the excitation light is assumed to be absorbed only by the surface, thus one can adopt the Dirac delta function $Q(z)=2 \delta(z)$.

The temperature gradients in the fluid and in the sample can be written in terms of the inverse of Laplace and Hankel transforms as

$$
T_{i}(r, z, t)=\int_{0}^{\infty} T_{i}(\alpha, z, t) \mathrm{J}_{0}(\alpha r) \alpha \mathrm{d} \alpha,
$$

where $\mathrm{J}_{0}(x)$ is the Bessel function of the first kind and

$$
T_{i}(\alpha, z, t)=\frac{k_{s} Q_{0} Q(\alpha)}{\sqrt{D_{s} / D_{f}}} \int_{t_{0}}^{t} G(\alpha, t-\tau) H\left(\alpha, \tau, D_{i}\right) \mathrm{d} \tau,
$$

in which $G(\alpha, t)$ and $H\left(\alpha, t, D_{i}\right)$ are given by the inverse Laplace transform of $G(\alpha, s)$ and $H\left(\alpha, s, D_{i}\right)$, respectively, as

$$
H\left(\alpha, t, D_{i}\right)=\left\{\begin{array}{cc}
\delta(t), & z=0, \\
\frac{|z| \exp \left[-D_{i} \alpha^{2} t-z^{2} /\left(4 D_{i} t\right)\right]}{\sqrt{4 \pi t^{3} D_{i}}}, & z \neq 0
\end{array}\right.
$$

and

$$
\begin{aligned}
G(\alpha, t)= & \frac{k_{s} \sqrt{D_{f}}}{\alpha \kappa\left(k_{s}^{2} D_{f}-k_{f}^{2} D_{s}\right)}\left[\sqrt{D_{s}} \operatorname{Erf}\left(\alpha \sqrt{D_{s}} \sqrt{t}\right)\right. \\
& \left.-e^{-\alpha^{2} \kappa t} \sqrt{D_{s}-\kappa} \operatorname{Erf}\left(\alpha \sqrt{D_{s}-\kappa} \sqrt{t}\right)\right] \\
& -\frac{k_{f} \sqrt{D_{s}}}{\alpha \kappa\left(k_{s}^{2} D_{f}-k_{f}^{2} D_{s}\right)}\left[\sqrt{D_{f}} \operatorname{Erf}\left(\alpha \sqrt{D_{f}} \sqrt{t}\right)\right. \\
& \left.-e^{-\alpha^{2} \kappa t} \sqrt{D_{f}-\kappa} \operatorname{Erf}\left(\alpha \sqrt{D_{f}-\kappa} \sqrt{t}\right)\right] .
\end{aligned}
$$

Here, $\kappa=\left(k_{s}^{2}-k_{f}^{2}\right) D_{s} D_{f} /\left(k_{s}^{2} D_{f}-k_{f}^{2} D_{s}\right), \operatorname{Erf}(x)$ is the error function, and $Q(\alpha)=\left(w_{\mathrm{e}}^{2} / 4\right) \exp \left(-w_{\mathrm{e}}^{2} \alpha^{2} / 8\right)$ is the Hankel transform of
$Q(r) . t_{0}=0$ accounts for the laser-on $(0<t<\xi)$ and $t_{0}=t-\xi$ for the laser-off $(t>\xi)$ excitation regimes. Both the excitation and the relaxation regimes are recorded experimentally. Using the null heat-flux approximation, $k_{f} \ll k_{s}$, the temperature change, on the surface at $z=0$, is reduced to

$$
T_{s}(\alpha, 0, t)=\frac{Q_{0} Q(\alpha)}{D_{s} \alpha} \operatorname{Erf}\left[\alpha \sqrt{D_{s} t}\right] .
$$

\section{Displacement field}

The temperature distribution within the solid is used as the source to compute the thermoelastic displacement of the sample by solving the thermoelastic equation of motion. The displacement field $\mathbf{u}$ is given by the solution of Ref. 38,

$$
(\lambda+2 \mu) \nabla^{2} \mathbf{u}+(\lambda+\mu) \nabla(\nabla \cdot \mathbf{u})=\gamma \nabla T_{s}(r, z, t)+\rho_{s} \frac{\partial^{2} \mathbf{u}}{\partial t^{2}},
$$

with free surface boundary conditions of the normal stress components in the semi-infinite plane, $\left.\sigma_{r z}\right|_{z=0}$ and $\left.\sigma_{z z}\right|_{z=0}$. Here, $\lambda=E v /[(1+v)(1-2 v)]$ and $\mu=E / 2(1+v)$ are the Lame's constants, $\gamma=(3 \lambda+2 \mu) \alpha_{T}, E$ is the Young's modulus, $\alpha_{T}$ is the linear thermal expansion coefficient, and $v$ is the Poisson's ratio of the sample. The last term on the right-hand side of Eq. (7) represents the inertia term yielding an elastic wave motion. This term can be neglected $^{37}$ as the elastic waves described by the inertia term are not observed experimentally since the detector averages them out due to its relatively long response time. ${ }^{35}$ The normal component of the displacement vector at the sample surface, $u_{z}(r, z=0, t)$, in null flux approximation, can be written for the semi-infinite sample as

$$
u_{z}(r, 0, t)=-2(1+v) \alpha_{T} \int_{0}^{\infty} \alpha^{2} f(\alpha, t) J_{0}(\alpha r) \mathrm{d} \alpha
$$

where

$$
f(\alpha, t)=Q_{0} Q(\alpha) \int_{t_{0}}^{t} \frac{1}{\alpha} \operatorname{Erfc}\left(\alpha \sqrt{D_{s} \tau}\right) \mathrm{d} \tau .
$$

For $r=0$, Eq. (8) reduces to

$$
u_{z}(0,0, t)=\theta \frac{\lambda_{p}}{2 \pi} \frac{2}{t_{c}} \int_{t_{0}}^{t}\left(1-\frac{1}{\sqrt{1+t_{c} / 2 \tau}}\right) \mathrm{d} \tau,
$$

where $t_{c}=w^{2} / 4 D_{s}, \theta=-P(1-R)(1+v) \alpha_{T} \phi /\left(k_{s} \lambda_{p}\right)$, and $\lambda_{p}$ is the wavelength of the probe beam. The effect of neglecting the heat-coupling in the determination of the surface displacement is discussed in Ref. 37.

\section{B. Finite element analysis (FEA)}

The heat transfer and thermoelastic equations, Eqs. (1) and (7), were also solved using FEA with Comsol Multiphysics 4.2a software, which provides numerical solutions to the differential equations with the realistic boundary conditions imposed by the experiments. 
The temperature and the displacement obtained with FEA are compared with the analytical solutions, Eqs. (2) and (8). We assumed that the mechanical and thermal properties of the sample do not change much as the temperature rise induced by the excitation laser is not large.

\section{Optical phase difference (OPD)}

The surface deformation of the sample and the change of the refractive index of the air $n_{f}(r, z, t)$ surrounding the sample due to heat coupling can be simultaneously measured by the interferometer. This information is encoded in the OPD generated between the two arms of the interferometer. The combined OPD is

$$
\Phi(r, t)=\Phi_{s}(r, t)+\Phi_{f}(r, t) .
$$

The first contribution

$$
\Phi_{s}(r, t)=\frac{4 \pi}{\lambda_{p}} u_{z}(r, 0, t)
$$

is the OPD induced by the normal thermoelastic deformation of the sample surface given by Eq. (8). The second contribution

$$
\Phi_{f}(r, t)=\frac{4 \pi}{\lambda_{p}} \frac{d n_{f}}{d T} \int_{-\infty}^{0} T_{f}(r, z, t) \mathrm{d} z
$$

is an additional OPD the interferometer senses as a consequence of the heating of the surrounding air, ${ }^{34}$ where $T_{f}(r, z, t)$ is given by Eqs. (2) and (3). Here, the temperature coefficient of the fluid refractive index at $\lambda_{p}, d n_{f} / d T$, is assumed temperature independent. Since $d n_{f} / d T$ for the air at normal conditions is negative, heating the air lowers its refractive index. The probing beam, therefore, propagates faster through the heated air that effectively shortens the time needed for the probing beam to return back and interfere with the reference beam. The same happens when the sample surface moves toward the incoming probing beam. Thus, the OPD induced due to air heating in front of the sample adds to the OPD created by the bulge due to thermal expansion.

The interferometer gives direct access to the time-varying OPD, also called a phase shift, at a selected off-axis distance $r$. The physical parameters of the sample and the fluid in $\Phi(r, t)$ can be rearranged in terms of $\theta$.

\section{EXPERIMENTAL}

\section{A. Samples}

Metals of different geometries were used to compare the numerical predictions with the interferometric experiments. Commercial grade (purity of 99\%) copper (C10100) and inox (310S) metals were prepared in cylindrical shapes with one flat surface polished with polycrystalline diamond compound in order to create a high reflective surface. Copper samples were $50.8 \mathrm{~mm}$ and $12.7 \mathrm{~mm}$ in diameter and $30 \mathrm{~mm}$ thick. Inox samples were $50.8 \mathrm{~mm}$ and $12.7 \mathrm{~mm}$ in diameter and $15 \mathrm{~mm}$ thick. The optical reflection coefficients $R$ of the samples were determined by measuring the incident and reflected power at the excitation wavelength and used to correct the absorbed power by the samples.

\section{B. Excitation}

The experimental setup is illustrated in Fig. 2. The samples were excited by a cw Gaussian TEM $_{00}$ laser beam (Coherent, Verdi G7, OPSL at $532 \mathrm{~nm}$ ) arranged practically colinear to the measurement arm of the interferometer so that the angle between the excitation and the probe beams was $<1^{\circ}$. The mechanical shutter (SRS, Model SR470) was placed at the focus of lens $\mathrm{L}_{2}$ and used to control the excitation exposure time $(\xi)$, i.e., the laser-on/off transients. The rise/fall time was approximately $7 \mu$ s. Lens $\mathrm{L}_{3}$ was placed to recollimate the beam. A small amount of the excitation light was then extracted by the beam sampler (BS) and sent to the photodiode PD (Trigger) (Thorlabs, Model DET10A/M) to trigger the digital oscilloscope (Tektronix, Model DPO 4102B) that recorded the data. Lens $\mathrm{L}_{4}$ was used to focus the excitation beam at the sample surface with a radius $w_{\mathrm{e}}=646 \mu \mathrm{m}$. The laser beams radii at the sample surface position were measured with a beam profiler (Thorlabs, Model BP104-UV).

\section{Detection}

The HQLI was employed to monitor the magnitude of the thermal expansion and the effect of TL above the sample due to laser excitation. It uses a stabilized and linear polarized $\mathrm{He}-\mathrm{Ne}$

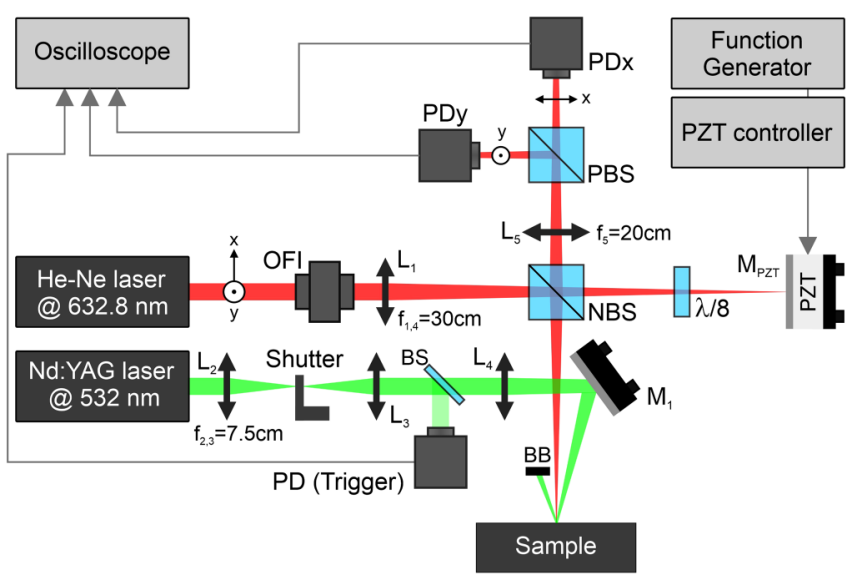

FIG. 2. Schematic illustration of the HQLI interferometer. The system is probed by a stabilized and linear polarized He-Ne laser at $632.8 \mathrm{~nm}$ with polarization perpendicular to the table, with polarization rotated by $45^{\circ}$ by the optical Faraday isolator (OFI). $L_{j}$ are the biconvex lenses of focal lengths $f_{j}, M_{j}$ are the mirrors, BB is the beam blocker, BS is the beam sampler, NBS/PBS are the nonpolarizing/polarizing beam splitters, $\lambda / 8$ is the octadic-wave plate and PDx,y are the photodiodes that detect the $x, y$-polarization of the laser, whose signals are acquired by the digital oscilloscope. The reference mirror $\left(M_{P Z T}\right)$ is attached to the piezoelectric transducer (PZT) controlled by the function generator. The thermoelastic displacement is induced in the sample by the continuous wave Gaussian beam in the fundamental mode $\mathrm{TEM}_{00}$ by an optically pumped semiconductor laser. The radii of the excitation and probe beams at the surface of the sample are: $w_{\mathrm{e}}=646 \mu \mathrm{m}$ and $w_{\text {int }}=154 \mu \mathrm{m}$, respectively. 
laser at $632.8 \mathrm{~nm}$ (Thorlabs, Model HRS015B, $1.2 \mathrm{~mW}$ ), with beam polarization perpendicular to the optical table. The optical Faraday isolator (OFI) rotates the polarization by $45^{\circ}$ and blocks any backreflections, preventing the laser to destabilize. Lens $\mathrm{L}_{1}$ focuses the beam at the sample surface with a radius of $w_{\text {int }}=154 \mu \mathrm{m}$. The non-polarizing beam splitter (NBS) splits the beam into the interferometer's measurement (reflected) and reference (transmitted) arms, evenly.

The beam in the reference arm is shifted by $90^{\circ}$ as it passes twice through the octadic-wave plate $(\lambda / 8)$, due to reflection from the reference mirror $\left(\mathrm{M}_{\mathrm{PZT}}\right)$, which can be displaced by the attached piezoelectric transducer (PZT) controlled by the function generator (Tektronix, Model AFG1022). The beam in the measurement arm is reflected and experiences a phase shift due to (i) the displacement of the sample surface due to laser-induced thermal expansion and (ii) the TL generated in the surrounding air. The two beams recombine and travel toward the polarizing beam splitter (PBS). In order to maintain the same wavefront curvature at the superposition of the two beams, the arms have to be of equal length, $20 \mathrm{~cm}$ in the present study. The orthogonal projection in the $\mathrm{x}$ direction ( $\mathrm{y}$ direction) is transmitted (reflected) and reaches the Si photodiode PDx (PDy). The two photodiodes are identical (Thorlabs, Model PDA36A-EC) and their raw output signals are acquired by the digital oscilloscope. As the beams recombine while diverging, lens $\mathrm{L}_{5}$ was placed to ensure that the photodiodes collect all the light.

\section{HQLI signal conditioning}

Consider ideal optical components, identical photodiodes with a linear response and the two ideal voltage signals $x_{i}$ and $y_{i}$ of the HQLI in perfect quadrature, i.e., with a relative phase shift of $90^{\circ}$ between the photodiode signals. In this case, the two ideal signals are given by ${ }^{31}$

$$
\begin{gathered}
x_{i}(t)=\frac{A_{0}}{4}(1+\sin \Phi(t)), \\
y_{i}(t)=\frac{A_{0}}{4}[1+\sin (\Phi(t)+\pi / 2)] \\
=\frac{A_{0}}{4}(1+\cos \Phi(t)),
\end{gathered}
$$

where $A_{0}$ stands for the voltage output if all laser light is collected by one photodiode. The optical phase difference (OPD) $\Phi(t)$, also simply dubbed the phase shift, given theoretically in Eq. (11), is the sum of the OPD caused by the out-of-plane thermal expansion [Eq. (12)] and the OPD induced by the TL [Eq. (13)]. Solving Eqs. (14), the phase shift is

$$
\Phi(t)=\arctan \left(\frac{x_{i}(t)-A_{0} / 4}{y_{i}(t)-A_{0} / 4}\right)+m \pi,
$$

where $m=0, \pm 1, \pm 2, \ldots$ must be chosen with a proper unwrapping algorithm to ensure the correction of the discontinuities of the arc-tangent function. By removing the DC offsets and equalizing the $\mathrm{AC}$ amplitudes to $A$, Eq. (14) can be rewritten as

$$
\begin{aligned}
& x_{i n}(t)=A \sin \Phi(t), \\
& y_{i n}(t)=A \cos \Phi(t) .
\end{aligned}
$$

Note the Lissajous curves of Eqs. (14) and (16) represent a perfect circle. The OPD is accessed by

$$
\Phi(t)=\arctan \left(\frac{x_{i n}}{y_{i n}}\right)+m \pi
$$

In the real experimental circumstances, the signals present nonlinearities despite of meticulous alignment. In this case, the raw signals assume the following form:

$$
\begin{gathered}
x(t)=x_{0}+A_{x} \sin \left(\Phi(t)+\Phi_{0}\right), \\
y(t)=y_{0}+A_{y} \cos \Phi(t) .
\end{gathered}
$$

The DC offsets $x_{0}$ and $y_{0}, \mathrm{AC}$ amplitudes $A_{x}$ and $A_{y}$, and the quadrature error $\Phi_{0}$ are known as common nonlinearities. ${ }^{27}$ The respective Lissajous curve is elliptically shaped as a result of different AC amplitudes, $A_{x} \neq A_{y}$, and the lack of quadrature, $\Phi_{0} \neq 0$.

The phase shift-Eq. (17) — when calculated directly from Eq. (18), presents second order periodic errors. ${ }^{27}$ The corrected phase, free of nonlinearities, is obtained by rearranging Eq. (18) in the form of Eq. (16), yielding

$$
\begin{aligned}
x_{c}(t)= & x_{r}(t)\left(\cos \Phi_{0}\right)^{-1}-y_{r}(t) \tan \Phi_{0} \\
& =\sin \Phi(t) \\
& y_{c}(t)=y_{r}(t)=\cos \Phi(t),
\end{aligned}
$$

where $x_{r}(t)$ and $y_{r}(t)$ are the reduced components given by the signals subtracted by the DC offsets and normalized by the AC amplitudes

$$
\begin{aligned}
& x_{r}(t)=\frac{x(t)-x_{0}}{A_{x}}, \\
& y_{r}(t)=\frac{y(t)-y_{0}}{A_{y}},
\end{aligned}
$$

respectively. Provided the nonlinearities are known, Eq. (19), therefore, leads to the corrected phase

$$
\Phi(t)=\arctan \left(\frac{x_{r}(t)}{y_{r}(t) \cos \Phi_{0}}-\tan \Phi_{0}\right)+m \pi .
$$

There are a few signal processing methods that allow for the determination of the five unknowns $\left(x_{0}, y_{0}, A_{x}, A_{y}, \Phi_{0}\right)$. Fitting an ellipse to the Lissajous curve is especially efficient, more so when the total displacement does not give a full ellipse. ${ }^{39}$ For convenience, in this paper, we made a synthetic displacement by vibrating the 
PZT in the reference arm of the HQLI to reach the maximum and minimum values of the interferometric signals before the measurements were performed. The latter approach was employed given it is less computationally consuming when dealing with large amounts of transients.

Interference signals of a synthetic displacement are shown in Fig. 3(a) and the Lissajous curve of the raw and corrected signals is shown in Fig. 3(b). Note how the ellipse has converted into a circle. Once the signal extremes are reached, the DC offsets and AC amplitudes can be extracted from the raw signals simply as

$$
\begin{aligned}
& \left(x_{0}, y_{0}\right)=\frac{\max (x, y)+\min (x, y)}{2}, \\
& \left(A_{x}, A_{y}\right)=\frac{\max (x, y)-\min (x, y)}{2} .
\end{aligned}
$$

The lack of quadrature $\Phi_{0}$ can be obtained from the extremes of the sum of the reduced signals $z=x_{r}+y_{r}$. The amplitude

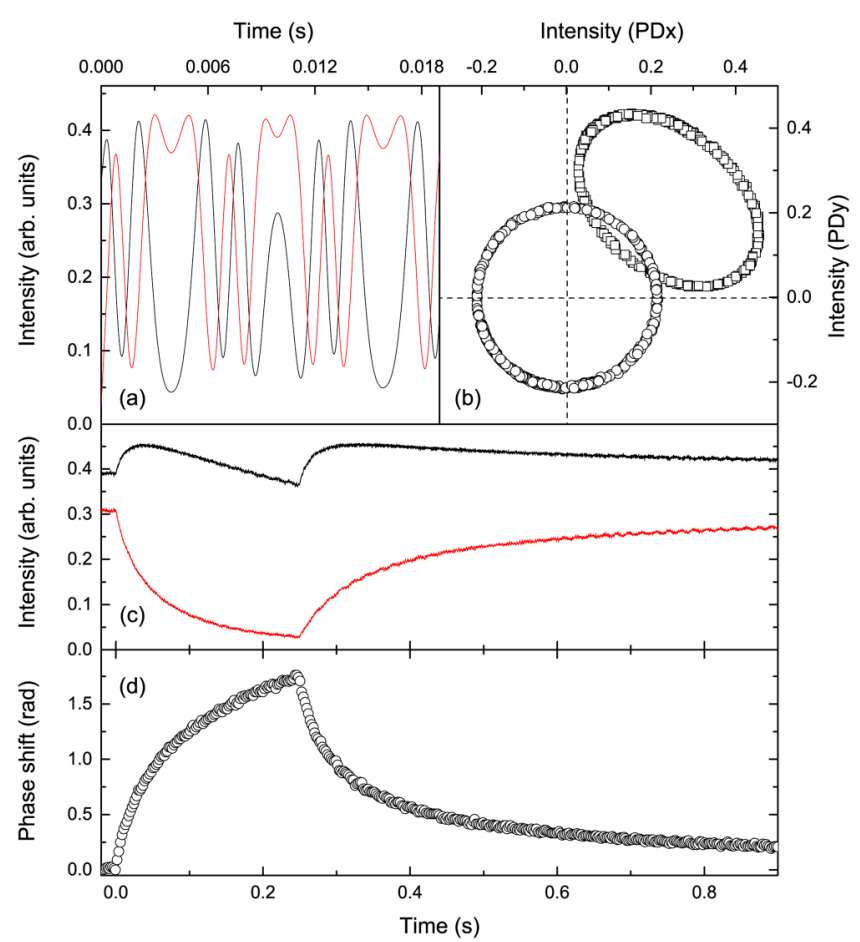

FIG. 3. Signal processing steps. (a) Synthetic interference signals produced by the piezoelectric transducer for determination of the common nonlinearities. (b) Lissajous curves from (a) corresponding to the raw (ellipse) and corrected (circle) signals. (c) Raw interference signals obtained from the laser-induced thermoelastic displacement and TL. Black/red line is the $x / y$-polarization signal detected by PDx/PDy. (d) Unwrapped phase transient of the signals from (c).
$A_{z}=(\max (z)-\min (z)) / 2$ is used to obtain the last nonlinearity

$$
\Phi_{0}=\arcsin \left(\frac{A_{z}^{2}-2}{2}\right)
$$

Raw interference signals of an example transient are shown in Fig. 3(c). Figure 3(d) exhibits the unwrapped phase transient.

\section{RESULTS AND DISCUSSION}

Following the laser-on/off experiment, each unwrapped interferometric transient is recorded and over 100 transients are averaged to produce the phase shift signal illustrated in Fig. 4 for copper with a diameter of $50.8 \mathrm{~mm}$ and an excitation power $P=2 \mathrm{~W}$. The exposure time $\xi=250 \mathrm{~ms}$ was used in this example. Continuous lines show the numerical curve fit using Eq. (11) (black). The agreement between the theory and the experiments is very good. The contributions to the phase shift from the surface displacement, $\Phi_{s}(0, t)$, and the thermal lens in the air, $\Phi_{f}(0, t)$, are also presented. The thermal lens in the air contributes around $4 \%$ to the total phase shift. The surface displacement $u_{z}(0,0, t)=\left(\lambda_{p} / 4 \pi\right) \Phi_{s}(0, t)$ is also shown on the right-hand side scale. Note that the peak displacement at the center of the excitation beam is less than $30 \mathrm{~nm}$.

Additional experiments were performed using different excitation times $\xi$, and the results for $\xi=20 \mathrm{~ms}$ and $\xi=250 \mathrm{~ms}$ are displayed as a function of the reduced time $t / \xi$ in Fig. 5 for copper with different diameters. The laser-off time between each excitation is long enough for the sample to return to its unperturbed thermal state. The samples were excited and probed with laser beam radii $w_{\mathrm{e}}=646 \mu \mathrm{m}$ and $w_{\text {int }}=154 \mu \mathrm{m}$, respectively, at the surface of the sample.

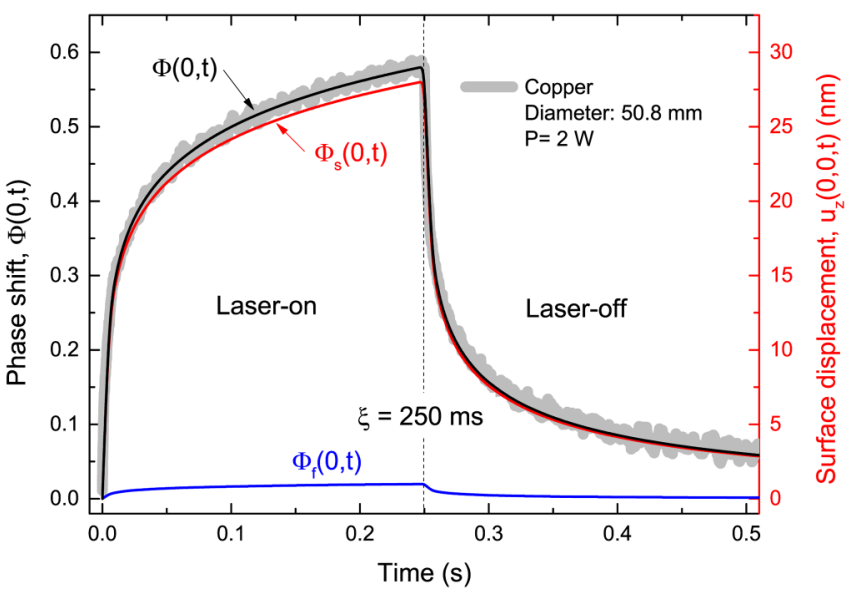

FIG. 4. Measured time-resolved interferometric phase shift for copper with a diameter of $50.8 \mathrm{~mm}$ and the excitation power $P=2 \mathrm{~W}$. Continuous lines show the numerical curve fit using Eq. (11) (black) and the contributions to the phase shift from the surface displacement (red) and the thermal lens in the air (blue). The surface displacement $u_{z}(0,0, t)=\left(\lambda_{p} / 4 \pi\right) \Phi_{s}(0, t)$ is also shown on the right-hand side scale. 


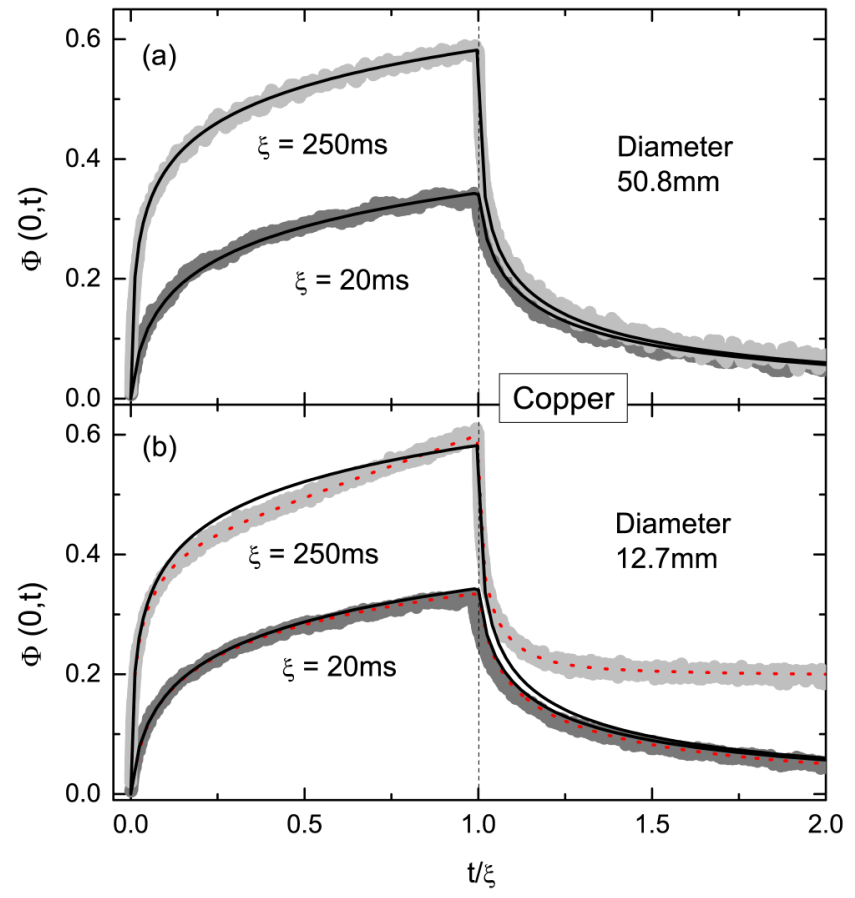

FIG. 5. Measured interferometric phase shift for copper with a diameter of (a) $50.8 \mathrm{~mm}$ and (b) $12.7 \mathrm{~mm}$ as a function of the reduced time, $t / \xi$. Continuous lines show the numerical fits of the curves in (a) using Eq. (11). Dotted lines (red) show the numerical predictions using the FEA with the parameters obtained from the fits in (a)

The continuous lines in Fig. 5 show the theoretical curve fits of the experimental phase shift transients for the sample with a diameter of $50.8 \mathrm{~mm}$-curves in (a) - to the analytical model, Eq. (11). The same curves are shown in (b) for the sample with a diameter of $12.7 \mathrm{~mm}$. The theoretical curves are in a good agreement with the experimental transients for the larger sample.

Using the same experimental configuration, a similar behavior was observed for the inox samples. Figure 6 shows the measured phase shifts for the samples with different diameters and exposure times from $\xi=100 \mathrm{~ms}$ to $\xi=5000 \mathrm{~ms}$. The time axis is presented in a $\log$ scale. The excitation power for the measurements was $P=600 \mathrm{~mW}$. The continuous lines displayed in Figs. 6(a) and 6(b) show the theoretical curve fits of the experimental phase shift transients for the sample with a diameter of $50.8 \mathrm{~mm}$ to Eq. (11). When the theoretical phase shifts for the samples with a diameter of $50.8 \mathrm{~mm}$ are projected over the experimental phase shifts for the samples with a diameter of $12.7 \mathrm{~mm}$, Fig. 5(b) for copper and Fig. 6(b) for inox, the curves are in disagreement with the experimental data, especially as the exposure time increases, both for copper $(\xi=250 \mathrm{~ms})$ and inox $(\xi>450 \mathrm{~ms})$. This is a clear evidence of the edge effects, or the lateral heat accumulation, contributing to the thermoelastic deformation of the samples and the consequent additional phase shift.

The thermal diffusivity and the parameter $\theta / P$ are retrieved from the regression analysis. Considering only the numerical fits

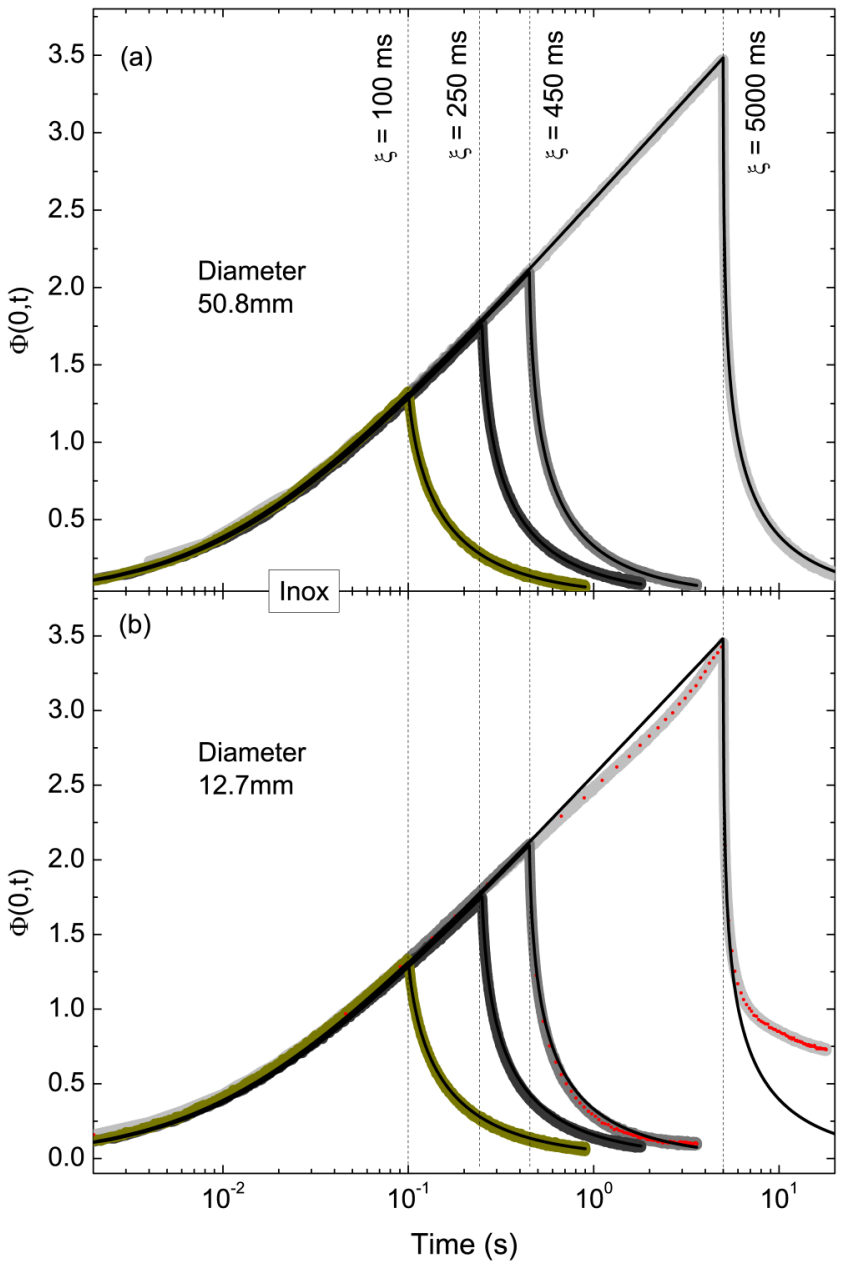

FIG. 6. Measured interferometric phase shift for inox with a diameter of (a) $50.8 \mathrm{~mm}$ and (b) $12.7 \mathrm{~mm}$ under different excitation time exposure ( $\xi$ ). Continuous lines show the numerical fits of the curves in (a) using Eq. (11). Time is shown on a log scale. Dotted lines (red) show the numerical predictions using FEA with the parameters obtained from the fits in (a).

over the transients for the samples with larger diameters, the results for the thermal diffusivity are within the range of expected values for both copper and inox, ${ }^{40,41}$ as shown in Table I. For copper, for instance, $D$ ranges from $116 \times 10^{-6} \mathrm{~m}^{2} \mathrm{~s}^{-1}$ for pure copper to $101 \times 10^{-6} \mathrm{~m}^{2} \mathrm{~s}^{-1}$ for copper alloy C15500. ${ }^{40}$ Assuming the values of $\alpha_{\mathrm{T}}$ and $v$ from the literature and the parameter $\theta$ measured in this study, the thermal conductivity was calculated and the results for both samples are also in good agreement with the previously reported data. ${ }^{34}$

The edge effects were considered in the experimental results by describing the dynamics of the systems using the FEA numerical calculations. The real geometry of the samples and the sample holders were assumed in the calculations. Figure 7 shows the temperature rise and the thermoelastic deformation calculated for inox 
TABLE I. Physical properties of the metals. Reflectivity $R$ is 0.58 for copper and 0.60 for inox.

\begin{tabular}{|c|c|c|c|c|c|c|}
\hline Material & $\begin{array}{c}D_{i} \\
\left(10^{-6}\right) \\
\mathrm{m}^{2} \mathrm{~s}^{-1}\end{array}$ & $\begin{array}{l}\theta / P \\
\mathrm{~W}^{-1}\end{array}$ & $\begin{array}{c}k_{i} \\
\mathrm{Wm}^{-1} \mathrm{~K}^{-1}\end{array}$ & $\begin{array}{c}\rho c_{p s} \\
\left(10^{6}\right) \\
\mathrm{Jm}^{-3} \mathrm{~K}^{-1}\end{array}$ & $v$ & $\begin{array}{c}\alpha_{T} \\
\left(10^{-6}\right) \\
\mathrm{K}^{-1}\end{array}$ \\
\hline Copper & $105 \pm 6$ & $0.042 \pm 0.001$ & $355 \pm 15$ & $3.4 \pm 0.2$ & $0.31^{a}$ & $17.3^{a}$ \\
\hline Inox & $3.6 \pm 0.1$ & $0.93 \pm 0.02$ & $15.2 \pm 0.7$ & $4.2 \pm 0.2$ & $0.29^{b}$ & $17.3^{b}$ \\
\hline \multicolumn{7}{|c|}{$d n_{\mathrm{air}} / d T=-1 \times 10^{-6} \mathrm{~K}^{-1 \mathrm{c}}$} \\
\hline
\end{tabular}

${ }^{a}$ Reference 40 .

${ }^{b}$ Reference 41 .

${ }^{c}$ Reference 42 .

(a)
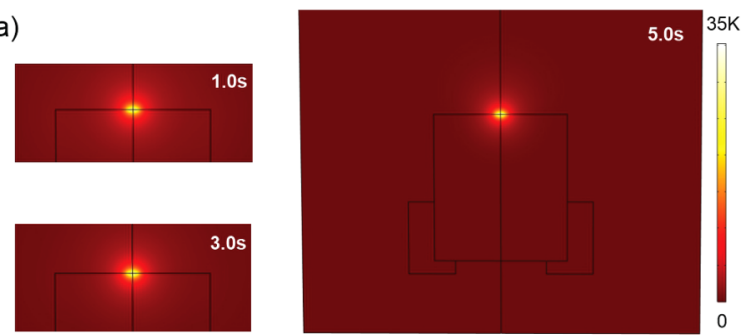

(b)
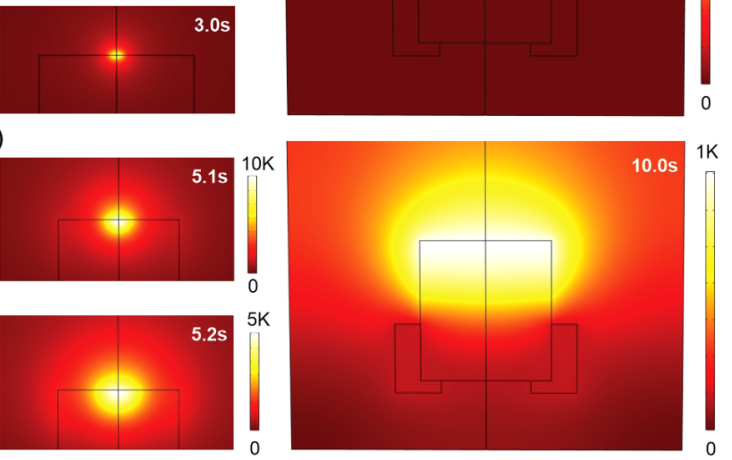

(c)

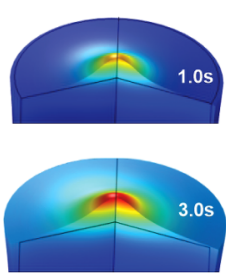

(d)
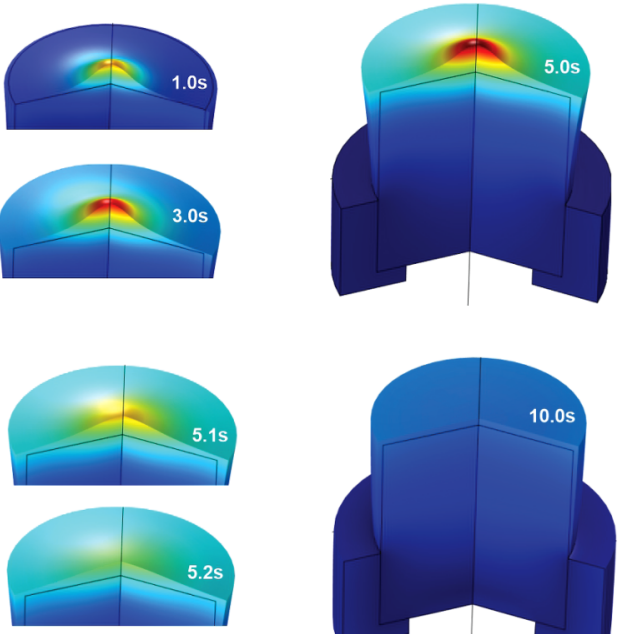

$700 \mathrm{~nm}$ $600 \mathrm{~nm}$
$500 \mathrm{~nm}$

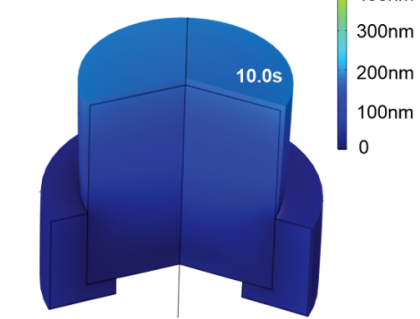

FIG. 7. Time evolution of the temperature rise and the thermoelastic displacement for laser-on (a) and (c) and laser-off (b) and (d) excitation of inox with a diameter of $12.7 \mathrm{~mm}$ with the excitation exposure time of $5 \mathrm{~s}$. with a diameter of $12.7 \mathrm{~mm}$ and the thickness of $15 \mathrm{~mm}$. The sample holder, made of aluminum, is also illustrated on the same scale. The simulations were performed using the same excitation power as in the experiments and the physical parameters as given in Table I. The excitation exposure time was $5 \mathrm{~s}$.

The edge effects are not clearly seen during the time the sample is excited (laser-on). However, when the laser is turned off, heat diffused rapidly throughout the material and reaches the boundaries of the samples. The heat is also accumulated in the center of the sample where the interferometer senses the phase shift. The heat-coupling between the sample and the surrounding air also contributes to the total phase shift observed experimentally. Analogously, the corresponding thermoelastic deformation of about $700 \mathrm{~nm}$ is observed at the center of the excitation beam in the sample during the laser-on excitation. The all-numerical results presented for inox in Fig. 7 was also calculated for copper (not shown here). These simulations were used to calculate the phase shift considering not only the real geometry of the samples, but also the finite size of the interferometer probe beam. The dotted lines (red) in Figs. 5(b) and 6(b) show the
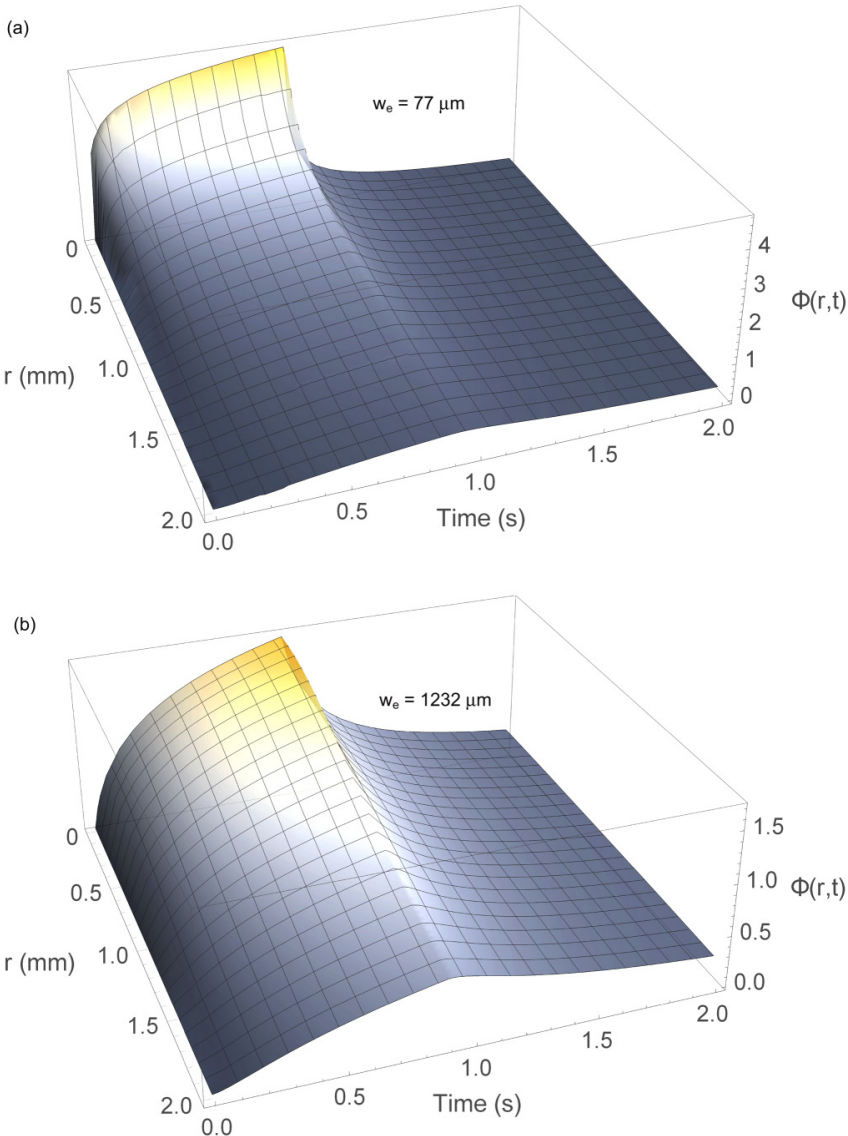

FIG. 8. FEA numerical calculations of the phase shift $\Phi(r, t)$ for inox with $\xi=900 \mathrm{~ms}$ as a function of $r$ for different excitation laser radius (a) $w_{\mathrm{e}}=77 \mu \mathrm{m}$ and (b) $w_{\mathrm{e}}=1232 \mu \mathrm{m}$. 
numerical FEA phase shifts for copper and inox, respectively, with a diameter of $12.7 \mathrm{~mm}$. There is an excellent agreement between the experiments and the numerical solutions for both samples.

With numerical calculations, the phase shift can be further explored as a function of the excitation beam radius used in the experiments. Figure 8 shows how the time-resolved phase shift is dispersed radially on the surface of inox (a diameter of $12.7 \mathrm{~mm}$ and the exposure time of $900 \mathrm{~ms}$ ). In Fig. 8(a), the shorter excitation beam radius generates a sharp response in time and a larger phase shift, attenuating further from the excitation origin. However, in Fig. 8(b), the phase shift is prolonged in time and along the surface of the sample as a result of the larger excitation spot.

Although the theoretical phase shift calculated so far assumes an infinitely small probe beam radius capable of probing at $r=0$, the real experiments are performed with a finite size probe beam radius ( $w_{\text {int }}=154 \mu \mathrm{m}$ in this study). The finite dimensions of the probe beam can be described by a theoretical averaged phase shift $\Phi_{\text {avg }}(t)$ considering the Gaussian radial distribution of the interferometer probe beam as

$$
\Phi_{\text {avg }}(t)=\frac{\int_{0}^{\infty} \Phi(r, t) \exp \left(-2 r^{2} / w_{\text {int }}^{2}\right) \mathrm{d} r}{\int_{0}^{\infty} \exp \left(-2 r^{2} / w_{\text {int }}^{2}\right) \mathrm{d} r} .
$$

Figure 9 shows the effects of the excitation beam radius on the phase shift as probed by the interferometer considering an infinitely small beam radius and the real finite size of the probe beam. The lines show $\Phi(0, t)$ considering different excitation beam radii, varying from $w_{\mathrm{e}}=77 \mu \mathrm{m}$ to $w_{\mathrm{e}}=1232 \mu \mathrm{m}$, compared with the respective averaged phase shift (symbols), $\Phi_{\text {avg }}(t)$, as probed by an interferometer with $w_{\text {int }}=154 \mu \mathrm{m}$. The inset presents the relative error between the phase shifts $\Phi(0, \xi) / \Phi_{\text {avg }}(\xi)$ as a function of the

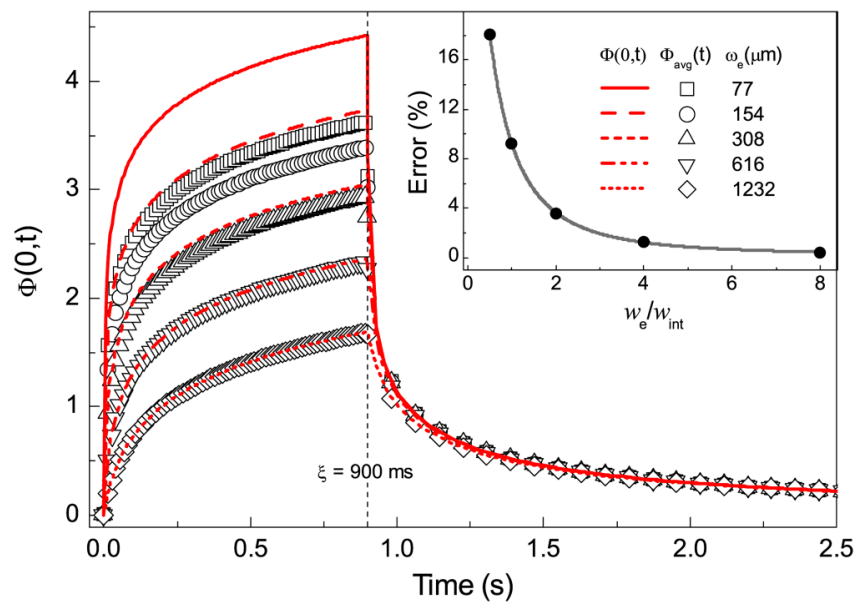

FIG. 9. FEA numerical calculations of the phase shift $\Phi(0, t)$ for inox with $\xi=$ $900 \mathrm{~ms}$ as a function of the excitation laser beam radius, varying from $77 \mu \mathrm{m}$ to $1232 \mu \mathrm{m}$, compared with the averaged phase shift $\Phi_{\text {avg }}(t)$, Eq. (24), as probed experimentally by the interferometer with a radius of $w_{\text {int }}=154 \mu \mathrm{m}$. The inset shows the relative error between the phase shifts $\Phi(0, \xi) / \Phi_{\text {avg }}(\xi)$ as a function of the ratio between the excitation and probe beam radii. ratio between the excitation and the probe beam radii. Note that the error decreases significantly as the ratio $w_{\mathrm{e}} / w_{\text {int }}$ increases.

\section{CONCLUSION}

To summarize, we have presented a detailed theoretical and experimental description of the edge effects on the photothermalinduced thermoelastic displacement of opaque metals detected by a homodyne quadrature laser interferometer. Both the excitation and the relaxation heat diffusion were investigated and compared to the analytical and the all-numerical solutions for the heat diffusion and the thermoelastic equations describing the phase shift on the sample. The finite geometry of the samples was crucial in determining how the temperature was distributed across the material and how this affected the interferometer phase shift measurements. The optical path change due to the surface thermoelastic deformation and the thermal lens in the surrounding air was decoded from the interferometric signal using analytical and numerical tools. The boundary/edge effects were found to be relevant to properly describe the interferometric signals and the tools developed in this study were found to be applicable to describe not only the phase shift sensed by the interferometer but also to contribute to the photothermal-based technologies employing similar detection mechanisms.

\section{ACKNOWLEDGMENTS}

This study was supported by CAPES (Finance Code 001); Fundação Araucária; Companhia Paranaense de Energia Elétrica COPEL (Projeto P\&D No. 2866-0466/2017-Código ANEEL); and CNPq (Process Nos. 401160/2016-5 and 401460/2016-9). The authors also acknowledge the financial support from the Slovenian Research Agency (research core Funding No. P2-0392 and Project No. L2-9254).

\section{DATA AVAILABILITY}

The data that support the findings of this study are available from the corresponding author upon reasonable request.

\section{REFERENCES}

${ }^{1}$ S. E. Bialkowski, N. G. C. Astrath, and M. A. Proskurnin, Photothermal Spectroscopy Methods, 2nd ed. (Wiley, 2019).

${ }^{2}$ C. B. Scruby and L. E. Drain, Laser Ultrasonics: Techniques and Applications, 2nd ed. (Adam Hilger, Bristol, 1990).

${ }^{3}$ H. A. Nugroho, D. A. Khusna, and E. L. Frannita, "Detection and classification of breast nodule on ultrasound images using edge feature," Int. J. Adv. Soft Comput. Appl. 11, 64-80 (2019).

${ }^{4}$ A. Mishra, R. Yadav, and L. Kumar, "Edge detection through dynamic programming in ultrasound gray scale digital images," in Intelligent Computing in Engineering. Advances in Intelligent Systems and Computing, edited by V. Solanki, M. Hoang, Z. Lu, and P. Pattnaik (Springer, Singapore, 2020), Vol. 1125, pp. 177-185.

${ }^{5}$ D. P. Almond and S. K. Lau, "Edge effects and a method of defect sizing for transient thermography,” Appl. Phys. Lett. 62, 3369-3371 (1993).

${ }^{6}$ L. Su, X. Yu, K. Li, X. Yao, and M. Pecht, "Simulation and experimental verification of edge blurring phenomenon in microdefect inspection based on highfrequency ultrasound," IEEE Access 7, 11515-11525 (2019).

${ }^{7} \mathrm{H}$. Shen, J. Hu, and Z. Yao, "Analysis and control of edge effects in laser bending,” Opt. Lasers Eng. 48, 305-315 (2010). 
${ }^{8}$ J. Hu, H. Xu, and D. Dang, "Modeling and reducing edge effects in laser bending," J. Mater. Process. Technol. 213, 1989-1996 (2013).

${ }^{9}$ Y. Shi, C. Zhang, G. Sun, and C. Li, "Study on reducing edge effects by using assistant force in laser forming," J. Mater. Process. Technol. 227, 169-177 (2016).

${ }^{10} \mathrm{C}$. Lv, K. Wang, G. Gu, and Y. Pan, "Accurate full-edge detection and depth measurement of internal defects using digital speckle pattern interferometry," NDT \& E Int. 102, 1-8 (2019).

${ }^{11}$ C. Taudt, T. Baselt, B. Nelsen, H. Assmann, A. Greiner, E. Koch, and P. Hartmann, "Characterization of edge effects in precision low-coherence interferometry using broadband light sources," SPIE Proc. 10329, 1032932 (2017).

${ }^{12}$ H. A. Weiss, S. Steentjes, P. Tröber, N. Leuning, T. Neuwirth, M. Schulz, K. Hameyer, R. Golle, and W. Volk, "Neutron grating interferometry investigation of punching-related local magnetic property deteriorations in electrical steels,” J. Magn. Magn. Mater. 474, 643-653 (2019).

${ }^{13} \mathrm{~K}$. Ritter and $\mathrm{K}$. Thiele, "Characterization of edge effects in precision lowcoherence interferometry using broadband light sources," in Proceedings of the 7th International Conference on Fracture Fatigue and Wear, Lecture Notes in Mechanical Engineering, edited by M. A. Wahab (Springer, 2019).

${ }^{14} \mathrm{R}$. Rodríguez-Martín, I. Castro, I. Ocaña, and J. Martínez-Esnaola, "Use of electronic speckle pattern interferometry in the detection of fatigue failure in high strength steels," Eng. Fail. Anal. 17, 226-235 (2010).

${ }^{15} \mathrm{X}$. Peng, A. Asundi, Y. Chen, and Z. Xiong, "Study of the mechanical properties of Nd:YVO4 crystal by use of laser interferometry and finite-element analysis," Appl. Opt. 40, 1396 (2001).

${ }^{16}$ G. Goetz, T. Ling, T. Gupta, S. Kang, J. Wang, P. D. Gregory, B. H. Park, and D. Palanker, "Interferometric mapping of material properties using thermal perturbation," Proc. Natl Acad. Sci. U.S.A. 115, E2499-E2508 (2018).

${ }^{17}$ J. Li, L. Zhong, Q. Zhang, Y. Zhou, J. Xiong, J. Tian, and X. Lu, "Optical image hiding based on dual-channel simultaneous phase-shifting interferometry and compressive sensing," Appl. Phys. B Lasers Opt. 123, 4 (2017).

${ }^{18} \mathrm{~J}$. Li, J. Sheng Li, Y. Yang Pan, and R. Li, "Compressive optical image encryption," Sci. Rep. 5, 10374 (2015)

${ }^{19} \mathrm{C}$. Hairaye, F. Mermet, T. Engel, P. C. Montgomery, and J. Fontaine, "Functionalization of surfaces by ultrafast laser micro/nano structuring," J. Phys. Conf. Ser. 558, 012063 (2014).

${ }^{20}$ A. Leong-Hoï, R. Claveau, M. Flury, W. Uhring, B. Serio, F. Anstotz, and P. C. Montgomery, "Detection of defects in a transparent polymer with high resolution tomography using white light scanning interferometry and noise reduction," in Videometrics, Range Imaging, and Applications XIII, edited by F. Remondino and M. R. Shortis (SPIE, 2015), Vol. 9528.

${ }^{21}$ L. Pramatarova, E. Radeva, E. Pecheva, T. Hikov, N. Krasteva, R. Dimitrova, D. Mitev, P. Montgomery, R. Sammons, and G. Altankov, "On Biomimetics," in On Biomimetics, edited by L. Pramatarova (InTech, 2011).

${ }^{22}$ E. Pecheva, P. Montgomery, D. Montaner, and L. Pramatarova, "White light scanning interferometry adapted for large-area optical analysis of thick and rough hydroxyapatite layers," Langmuir 23, 3912-3918 (2007).

${ }^{23}$ A. Leong-Hoi, P. C. Montgomery, B. Serio, W. Uhring, and E. Pecheva, "Improvement in measurements of hydroxyapatite layers by hybrid high dynamic range image processing in white-light interferometry," Mater. Today. Proc. 4, S36-S43 (2017).
${ }^{24}$ J.-P. Monchalin, "Laser-ultrasonics: Principles and industrial applications," in Ultrasonic and Advanced Methods for Nondestructive Testing and Material Characterization (World Scientific, 2007).

${ }^{25}$ B. Dong, C. Sun, and H. F. Zhang, "Optical detection of ultrasound in photoacoustic imaging," IEEE Trans. Biomed. Eng. 64, 4-15 (2017).

${ }^{26}$ G. Wissmeyer, M. A. Pleitez, A. Rosenthal, and V. Ntziachristos, "Looking at sound: Optoacoustics with all-optical ultrasound detection," Light Sci. Appl. 7, 53 (2018).

${ }^{27} \mathrm{~T}$. Požar, P. Gregorčič, and J. Možina, "A precise and wide-dynamic-range displacement-measuring homodyne quadrature laser interferometer," Appl. Phys. B Lasers Opt. 105, 575-582 (2011).

${ }^{28}$ Bureau International des Poids et Mesures, "Appendix A," in The International System of Units (SI) (Bureau international des poids et mesures, 2019).

${ }^{29}$ G. A. Lenkova, A. I. Lokhmatov, E. I. Gurin, E. B. Koronkevich, V. P. Kotesova, and G. G. Tarasov, "Laser interferometer for length measurements," Meas. Tech. 14, 1822-1825 (1971).

${ }^{30}$ J. Lazar, M. Holá, O. Číp, J. Hrabina, and J. Oulehla, "Interferometric system with tracking refractometry capability in the measuring axis," Meas. Sci. Technol. 24, 067001 (2013)

${ }^{31} \mathrm{P}$. Gregorčič, T. Požar, and J. Možina, "Quadrature phase-shift error analysis using a homodyne laser interferometer," Opt. Express 17, 16322-16331 (2009).

${ }^{32}$ A. Pogačnik, T. Požar, M. Kalin, and J. Možina, "A homodyne quadrature laser interferometer for micro-asperity deformation analysis," Sensors 13, 703-720 (2013).

${ }^{33} \mathrm{~S}$. Svitlov and A. Araya, "Homodyne interferometry with quadrature fringe detection for absolute gravimeter,” Appl. Opt. 53, 3548-3555 (2014).

${ }^{34}$ G. A. S. Flizikowski, O. A. Capeloto, V. G. Camargo, B. Anghinoni, M. L. Baesso, L. C. Malacarne, M. P. Belançon, T. Požar, and N. G. C. Astrath, "Laser induced thermoelastic surface displacement in solids detected simultaneously by photothermal mirror and interferometry," Opt. Express 28, 7116-7124 (2020).

${ }^{35}$ J. B. Spicer and D. H. Hurley, "Epicentral and near epicenter surface displacements on pulsed laser irradiated metallic surfaces," Appl. Phys. Lett. 68, 3561-3563 (1996).

${ }^{36} \mathrm{H}$. S. Carslaw and J. C. Jaeger, Conduction of Heat in Solids, 2nd ed. (Oxford University Press, 1959).

${ }^{37}$ G. V. B. Lukasievicz, L. C. Malacarne, N. G. C. Astrath, V. S. Zanuto, L. S. Herculano, and S. E. Bialkowski, "A theoretical and experimental study of time-resolved thermal mirror with non-absorbing heat-coupling fluids," Appl. Spectrosc. 66, 1461-1467 (2012).

${ }^{38}$ W. Nowacki, Thermoelasticity, 2nd ed. (Pergamon Press, 1982).

${ }^{39}$ T. Požar and J. Možina, "Enhanced ellipse fitting in a two-detector homodyne quadrature laser interferometer," Meas. Sci. Technol. 22, 085301 (2011).

${ }^{40}$ ASM Handbook, Properties and Selection: Nonferrous Alloys and Special-Purpose Materials, 10th ed. (ASM International, Materials Park, OH, 1990), Vol. 2.

${ }^{41}$ ASM Handbook, Properties and Selection: Irons, Steels, and High-Performance Alloys, 10th ed. (ASM International, Materials Park, OH, 1990), Vol. 1.

${ }^{42}$ W. M. Haynes, D. R. Lide, and T. J. Bruno, CRC Handbook of Chemistry and Physics: A Ready-Reference Book of Chemical and Physical Data, 97th ed. (CRC Press, Boca Raton, FL, 2016). 